\title{
Synthesising Interprocedural Bit-Precise Termination Proofs
}

\author{
Hong-Yi Chen, Cristina David, Daniel Kroening, Peter Schrammel and Björn Wachter \\ Department of Computer Science, University of Oxford, first.lastname@cs.ox.ac.uk
}

\begin{abstract}
Proving program termination is key to guaranteeing absence of undesirable behaviour, such as hanging programs and even security vulnerabilities such as denial-of-service attacks. To make termination checks scale to large systems, interprocedural termination analysis seems essential, which is a largely unexplored area of research in termination analysis, where most effort has focussed on difficult single-procedure problems. We present a modular termination analysis for $\mathrm{C}$ programs using templatebased interprocedural summarisation. Our analysis combines a context-sensitive, over-approximating forward analysis with the inference of under-approximating preconditions for termination. Bit-precise termination arguments are synthesised over lexicographic linear ranking function templates. Our experimental results show that our tool $2 \mathrm{LS}$ outperforms state-of-the-art alternatives, and demonstrate the clear advantage of interprocedural reasoning over monolithic analysis in terms of efficiency, while retaining comparable precision.
\end{abstract}

\section{INTRODUCTION}

Termination bugs can compromise safety-critical software systems by making them irresponsive, e.g., termination bugs can be exploited in denial-of-service attacks [1]. Termination guarantees are therefore instrumental for software reliability. Termination provers, static analysis tools that aim to construct a termination proof for a given input program, have made tremendous progress. They enable automatic proofs for complex loops that may require linear lexicographic (e.g. [2], [3]) or non-linear termination arguments (e.g. [4]) in a completely automatic way. However, there remain major practical challenges in analysing real-world code.

First of all, as observed by [5], most approaches in the literature are specialised to linear arithmetic over unbounded mathematical integers. Although, unbounded arithmetic may reflect the intuitively-expected program behaviour, the program actually executes over bounded machine integers. The semantics of $\mathrm{C}$ allows unsigned integers to wrap around when they over/underflow. Hence, arithmetic on $k$-bit-wide unsigned integers must be performed modulo- $2^{k}$. According to the $\mathrm{C}$ standards, over/underflows of signed integers are undefined behaviour, but practically also wrap around on most architectures. Thus, accurate termination analysis requires a bit-precise analysis of program semantics. Tools must be configurable with architectural specifications such as the width of data types and endianness. The following examples illustrate that termination behaviour on machine integers can be completely different than on mathematical integers. For example, the following code:

void foo $1($ unsigned $\mathrm{n}) \quad\{$ for (unsigned $\mathrm{x}=0 ; \mathrm{x}<=\mathrm{n} ; \mathrm{x}++) ;\}$ does terminate with mathematical integers, but does not terminate with machine integers if $\mathrm{n}$ equals the largest unsigned integer. On the other hand, the following code:

void foo $2($ unsigned $\mathrm{x})\{$ while $(\mathrm{x}>=10) \mathrm{x}++$; $\}$

does not terminate with mathematical integers, but terminates with machine integers because unsigned machine integers wrap around.

A second challenge is to make termination analysis scale to larger programs. The yearly Software Verification Competition (SV-COMP) [6] includes a division in termination analysis, which reflects a representative picture of the state-of-the-art. The SV-COMP'15 termination benchmarks contain challenging termination problems on smaller programs with at most 453 instructions (average 53), contained at most 7 functions (average 3), and 4 loops (average 1).

In this paper, we present a technique that we have successfully run on programs that are one magnitude larger, containing up to 5000 instructions. Larger instances require different algorithmic techniques to scale, e.g., modular interprocedural analysis rather than monolithic analysis. This poses several conceptual and practical challenges that do not arise in monolithic termination analysers. For example, when proving termination of a program, a possible approach is to try to prove that all procedures in the program terminate universally, i.e., in any possible calling context. However, this criterion is too optimistic, as termination of individual procedures often depends on the calling context, i.e., procedures terminate conditionally only in specific calling contexts.

Hence, an interprocedural analysis strategy is to verify universal program termination in a top-down manner by proving termination of each procedure relative to its calling contexts, and propagating upwards which calling contexts guarantee termination of the procedure. It is too difficult to determine these contexts precisely; analysers thus compute preconditions for termination. A sufficient precondition identifies those prestates in which the procedure will definitely terminate, and is thus suitable for proving termination. By contrast, a necessary precondition identifies the pre-states in which the procedure may terminate. Its negation are those states in which the procedure will not terminate, which is useful for proving nontermination.

In this paper we focus on the computation of sufficient preconditions. Preconditions enable information reuse, and thus scalability, as it is frequently possible to avoid repeated analysis of parts of the code base, e.g. libraries whose procedures 
Definition 2 (Ranking function). $A$ ranking function for $a$ procedure (Init, Trans, Out) with invariant Inv is a function $r$ from the set of program states to a well-founded domain such that $\forall \boldsymbol{x}, \boldsymbol{x}^{\prime}: \operatorname{Inv}(\boldsymbol{x}) \wedge \operatorname{Trans}\left(\boldsymbol{x}, \boldsymbol{x}^{\prime}\right) \Longrightarrow r(\boldsymbol{x})>r\left(\boldsymbol{x}^{\prime}\right)$.

We denote by $R R\left(\boldsymbol{x}, \boldsymbol{x}^{\prime}\right)$ the constraints that guarantee that $r$ is a ranking function. The existence of a ranking function for a procedure guarantees its universal termination.

The weakest termination precondition for a procedure describes the inputs for which it terminates. If it is true, the procedure terminates universally; if it is false, then it does not terminate for any input. Since the weakest precondition is intractable to compute or even uncomputable, we underapproximate the precondition. A sufficient precondition for termination guarantees that the program terminates for all $x^{i n}$ that satisfy it.

Definition 3 (Precondition for termination). Given a procedure (Init, Trans, Out), a sufficient precondition for termination is a predicate Precond such that

$$
\begin{aligned}
& \exists R R, \text { Inv }: \forall \boldsymbol{x}^{i n}, \boldsymbol{x}, \boldsymbol{x}^{\prime}: \\
& \quad \operatorname{Precond}\left(\boldsymbol{x}^{i n}\right) \wedge \operatorname{Init}\left(\boldsymbol{x}^{i n}, \boldsymbol{x}\right) \Longrightarrow \operatorname{Inv}(\boldsymbol{x}) \\
& \wedge \quad \operatorname{Inv}(\boldsymbol{x}) \wedge \operatorname{Trans}\left(\boldsymbol{x}, \boldsymbol{x}^{\prime}\right) \Longrightarrow \operatorname{Inv}\left(\boldsymbol{x}^{\prime}\right) \wedge R R\left(\boldsymbol{x}, \boldsymbol{x}^{\prime}\right)
\end{aligned}
$$

Note that false is always a trivial model for Precond, but not a very useful one.

\section{OVERVIEW OF THE APPROACH}

In this section, we introduce the architecture of our interprocedural termination analysis. Our analysis combines, in a nontrivial synergistic way, the inference of invariants, summaries, calling contexts, termination arguments, and preconditions, which have a concise characterisation in second-order logic (see Definitions 1, and 3). At the lowest level our approach relies on a solver backend for second-order problems, which is described in Sec. V.

To see how the different analysis components fit together, we now go through the pseudo-code of our termination analyser (Algorithm 1). Function analyze is given the entry procedure $f_{\text {entry }}$ of the program as argument and proceeds in two analysis phases.

Phase one is an over-approximate forward analysis, given in subroutine analyzeForward, which recursively descends into the call graph from the entry point $f_{\text {entry }}$. Subroutine analyzeForward infers for each procedure call in $f$ an overapproximating calling context $\mathrm{CallCtx}^{\circ}$, using procedure summaries and other previously-computed information. Before analyzing a callee, the analysis checks if the callee has already been analysed and, whether the stored summary can be reused, i.e., if it is compatible with the new calling context CallCtx ${ }^{o}$. Finally, once summaries for all callees are available, the analysis infers loop invariants and a summary for $f$ itself, which are stored for later re-use by means of a join operator.

The second phase is an under-approximate backward analysis, subroutine analyzeBackward, which infers termination preconditions. Again, we recursively descend into the call graph. Analogous to the forward analysis, we infer for each

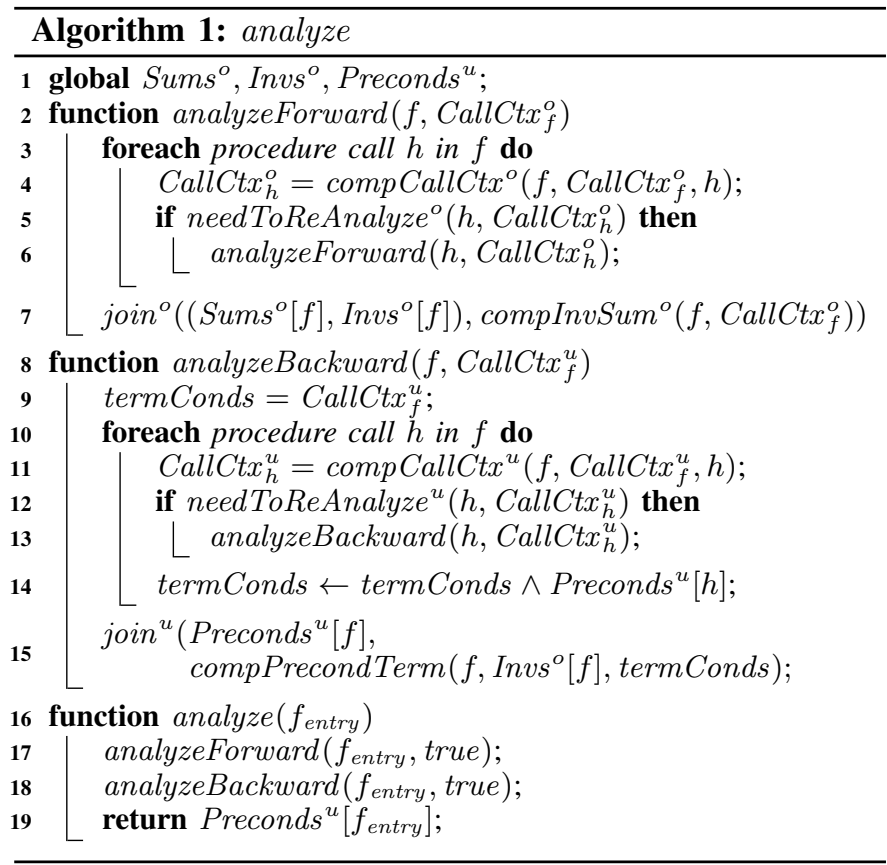

procedure call in $f$ an under-approximating calling context CallCtx $^{u}$ (using under-approximate summaries, as described in Sec. IV), and recurs only if necessary (Line 12). Finally, we compute the under-approximating precondition for termination (Line 15). This precondition is inferred w.r.t. the termination conditions that have been collected: the backward calling context (Line 9), the preconditions for termination of the callees (Line 14), and the termination arguments for $f$ itself (see Sec. IV). Note that superscripts $o$ and $u$ in predicate symbols indicate over- and underapproximation, respectively.

Challenges. Our algorithm uses over- and underapproximation in a systematic way in order to address the challenging problem of finding meaningful preconditions by a context-sensitive interprocedural termination analysis.

- The precondition in Definition 3 admits the trivial solution false for Precond. How do we find a good candidate? To this end, we "bootstrap" the process with a candidate precondition: a single value of $\boldsymbol{x}^{i n}$, for which we compute a termination argument. The key observation is that the resulting termination argument is typically more general, i.e., it shows termination for many further entry states. The more general precondition is then computed by precondition inference w.r.t. the termination argument.

- A second challenge is to compute under-approximations. Obviously, the predicates in the definitions in Sec. II can be over-approximated by using abstract domains such as intervals. However, there are only few methods for underapproximating analysis. In this work, we use a method similar to [8] to obtain under-approximating preconditions w.r.t. property $p$ : we infer an over-approximating precondition w.r.t. $\neg p$ and negate the result. In our case, $p$ is the termination condition termConds. 
Example 1. We illustrate the algorithm on the simple example given as Fig. 1 with the encoding in Fig. 2. f calls a procedure h. Procedure $h$ terminates if and only if its argument $y$ is non-zero, i.e., procedure f only terminates conditionally. The call of $h$ is guarded by the condition $z>0$, which guarantees universal termination of procedure $f$.

Let us assume that unsigned integers are 32 bits wide, and we use an interval abstract domain for invariant, summary and precondition inference, but the abstract domain with the elements $\{$ true, false $\}$ for computing calling contexts, i.e., we can prove that calls are unreachable. We use $M:=2^{32}-1$.

Our algorithm proceeds as follows. The first phase is analyzeForward, which starts from the entry procedure $f$. By descending into the call graph, we must compute an overapproximating calling context CallCtx $x_{h}^{o}$ for procedure $h$ for which no calling context has been computed before. This calling context is true. Hence, we recursively analyse h. Given that $h$ does not contain any procedure calls, we compute the over-approximating summary Sum $_{h}^{o}=\left(0 \leq y \leq M \wedge 0 \leq r_{h} \leq M\right)$ and invariant Inv $v_{h}^{o}=(0 \leq x \leq M \wedge 0 \leq y \leq M)$. Now, this information can be used in order to compute Sum $_{f}^{o}=$ $\left(0 \leq z \leq M \wedge 0 \leq r_{f} \leq M\right)$ and invariant Inv ${ }_{f}^{o}=$ true for the entry procedure $f$.

The backwards analysis starts again from the entry procedure f. It computes an under-approximating calling context CallCtx ${ }_{h}^{u}$ for procedure $h$, which is true, before descending into the call graph. It then computes an under-approximating precondition for termination Precond $d_{h}^{u}=(1 \leq y \leq M)$ or, more precisely, an under-approximating summary whose projection onto the input variables of $h$ is the precondition Precond $d_{h}^{u}$. By applying this summary at the call site of $h$ in $f$, we can now compute the precondition for termination Precond $d_{f}^{u}=$ $(0 \leq z \leq M)$ of $f$, which proves universal termination of $f$.

We illustrate the effect of the choice of the abstract domain on the analysis of the example program. Assume we replace the $\{$ true, false $\}$ domain by the interval domain. In this case, analyzeForward computes CallCtx $x_{h}^{o}=(1 \leq z \leq M \wedge$ $\left.0 \leq w_{1} \leq M\right)$. The calling context is computed over the actual parameters $z$ and $w_{1}$. It is renamed to the formal parameters $y$ and $r_{h}$ (the return value) when CallCtx $x_{h}^{o}$ is used for constraining the pre/postconditions in the analysis of h. Subsequently, analyzeBackward computes the precondition for termination of $h$ using the union of all calling contexts in the program. Since $h$ terminates unconditionally in these calling contexts, we trivially obtain Precond $d_{h}^{u}=(1 \leq y \leq M)$, which in turn proves universal termination of $f$.

\section{INTERPRocedURAL TERMINATION ANALYSIS}

We can view Alg. 1 as solving a series of formulae in second-order predicate logic with existentially quantified predicates, for which we are seeking satisfiability witnesses. ${ }^{3}$ In this section, we state the constraints we solve, including all the side constraints arising from the interprocedural analysis.

\footnotetext{
${ }^{3}$ To be precise, we are not only looking for witness predicates but (good approximations of ) weakest or strongest predicates. Finding such biased witnesses is a feature of our synthesis algorithms.
}

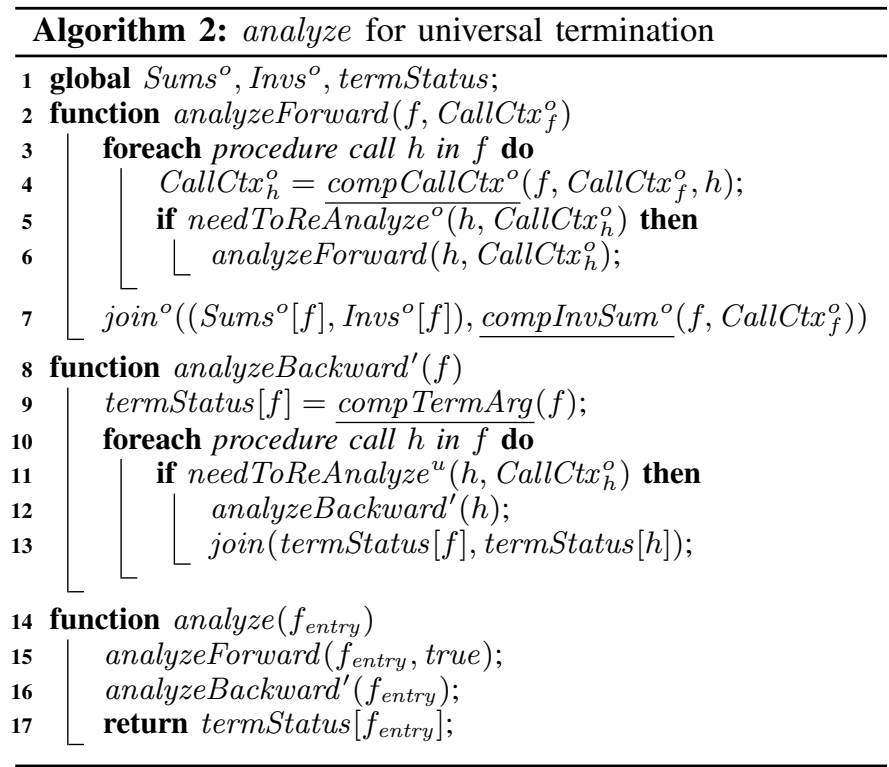

Note that this is not a formalisation exercise, but these are precisely the formulae solved by our synthesis backend, which is described in Section V.

\section{A. Universal Termination}

For didactical purposes, we start with a simplification of Algorithm 1 that is able to show universal termination (see Algorithm 2). This variant reduces the backward analysis to a call to compTermArg and propagating back the qualitative result obtained: terminating, potentially non-terminating, or non-terminating.

This section states the constraints that are solved to compute the outcome of the functions underlined in Algorithm 2 and establish its soundness:

- $\operatorname{compCallCtx}^{\circ}$ (Def. 4)

- compInvSum ${ }^{o}$ (Def. 5)

- compTermArg (Lemma 3)

Definition 4 (compCallCtx $\left.{ }^{\circ}\right)$. A forward calling context CallCtx $h_{h_{i}}^{o}$ for $h_{i}$ in procedure $f$ in calling context CallCtx ${ }_{f}^{o}$ is a satisfiability witness of the following formula:

$$
\begin{aligned}
& \exists \text { CallCtx }_{h_{i}}^{o}, \text { Inv }_{f}^{o}: \forall \boldsymbol{x}^{\text {in }}, \boldsymbol{x}, \boldsymbol{x}^{\prime}, \boldsymbol{x}^{\text {out }}, \boldsymbol{x}^{p_{-} \text {in }}{ }_{i}, \boldsymbol{x}^{p_{-} \text {out }}{ }_{i}: \\
& \text { CallCtx }{ }_{f}^{o}\left(\boldsymbol{x}^{\text {in }}, \boldsymbol{x}^{\text {out }}\right) \wedge \text { Summs }_{f}^{o} \Longrightarrow \\
& \left(\operatorname{Init}_{f}\left(\boldsymbol{x}^{i n}, \boldsymbol{x}\right) \Longrightarrow \operatorname{Inv}_{f}^{o}(\boldsymbol{x})\right) \\
& \wedge\left(\operatorname{Inv}_{f}^{o}(\boldsymbol{x}) \wedge \operatorname{Trans}_{f}\left(\boldsymbol{x}, \boldsymbol{x}^{\prime}\right)\right. \\
& \Longrightarrow \operatorname{Inv}_{f}^{o}\left(\boldsymbol{x}^{\prime}\right) \wedge\left(g_{h_{i}} \Rightarrow \operatorname{CallCtx} h_{h_{i}}^{o}\left(\boldsymbol{x}^{p_{-} \text {in }}{ }_{i}, \boldsymbol{x}^{p_{-} \text {out }}{ }_{i}\right)\right)
\end{aligned}
$$

with Summs $_{f}^{o}=\bigwedge_{\text {calls } h_{j} \text { in } f} \quad g_{h_{j}} \Longrightarrow$

$$
\text { Sums }^{o}[h]\left(\boldsymbol{x}_{-i n}^{p_{j}}, \boldsymbol{x}^{p_{-} \text {out }}{ }_{j}\right)
$$

where $g_{h_{j}}$ is the guard condition of procedure call $h_{j}$ in $f$ capturing the branch conditions from conditionals. For example, $g_{h_{0}}$ of the procedure call to $\mathrm{h}$ in $\mathrm{f}$ in Fig. 1 is $z>0$. Sums ${ }^{o}[h]$ is the currently available summary for $\mathrm{h}$ (cf. global variables in Alg. 1). 
Lemma 1. CallCtx $x_{h_{i}}^{o}$ is over-approximating.

Proof sketch. CallCtx $x_{f}^{o}$ when $f$ is the entry-point procedure is true; also, the summaries $S_{u m}^{o}{ }_{h_{j}}^{o}$ are initially assumed to be true, i.e. over-approximating. Hence, given that CallCtx ${ }_{f}^{o}$ and Summs $_{f}^{o}$ are over-approximating, CallCtx ${ }_{h_{i}}^{o}$ is overapproximating by the soundness of the synthesis (see Thm. 3 in Sec. V).

Example 2. Let us consider procedure $f$ in Fig. 1 . $f$ is the entry procedure, hence we have CallCtx ${ }_{f}^{o}\left((z),\left(r_{f}\right)\right)=$ true (= $\left(0 \leq z \leq M \wedge 0 \leq r_{f} \leq M\right)$ where $M:=2^{32}-1$ when using the interval abstract domain for 32 bit integers). Then, we instantiate Def. 4 (for procedure f) to compute CallCtx ${ }_{h_{0}}^{o}$. We assume that we have not yet computed a summary for h, thus, $S_{\text {Sum }}^{o}$ is true. Remember that the placeholder $h_{0}\left((z),\left(w_{1}\right)\right)$ evaluates to true.

$$
\begin{aligned}
& \exists \text { CallCtx }_{h_{0}}^{o}, \text { Inv }_{f}^{o}: \forall z, w_{1}, w, w^{\prime}, z^{\prime}, g, g^{\prime}, r_{f}: \\
& 0 \leq z \leq M \wedge 0 \leq r_{f} \leq M \wedge(z>0 \Longrightarrow \text { true }) \Longrightarrow \\
& \quad\left(w=0 \wedge z^{\prime}=z \wedge g \Longrightarrow \operatorname{Inv}_{f}^{o}((w, z, g))\right) \\
& \wedge\left(\operatorname{Inv}_{f}^{o}((w, z, g)) \wedge\right. \\
& \quad g \wedge h_{0}\left((z),\left(w_{1}\right)\right) \wedge w^{\prime}=\left(z>0 ? w_{1}: w\right) \wedge z^{\prime}=z \wedge \neg g^{\prime} \\
& \quad \Longrightarrow \operatorname{Inv}_{f}^{o}\left(\left(w^{\prime}, z^{\prime}, g^{\prime}\right)\right) \wedge\left(z>0 \Rightarrow \operatorname{CallCtx}_{h_{i}}^{o}\left((z),\left(w_{1}\right)\right)\right)
\end{aligned}
$$

A solution is Inv $_{f}^{o}=$ true, and CallCtx ${ }_{h_{0}}^{o}\left((z),\left(w_{1}\right)\right)=$ $\left(1 \leq z \leq M \wedge 0 \leq w_{1} \leq M\right)$.

Definition 5 (compInvSum $\left.{ }^{o}\right)$. A forward summary Sum $_{f}^{o}$ and invariants Inv $v_{f}^{o}$ for procedure $f$ in calling context CallCtx ${ }_{f}^{o}$ are satisfiability witnesses of the following formula:

$$
\begin{aligned}
& \exists \operatorname{Sum}_{f}^{o}, \operatorname{Inv}_{f}^{o}: \forall \boldsymbol{x}^{\text {in }}, \boldsymbol{x}, \boldsymbol{x}^{\prime}, \boldsymbol{x}^{\prime \prime}, \boldsymbol{x}^{\text {out }}: \\
& \quad \operatorname{CallCtx}_{f}^{o}\left(\boldsymbol{x}^{\text {in }}, \boldsymbol{x}^{\text {out }}\right) \wedge \operatorname{Summs}_{f}^{o} \Longrightarrow \\
& \quad\left(\operatorname{Init}_{f}\left(\boldsymbol{x}^{\text {in }}, \boldsymbol{x}\right) \wedge \operatorname{Inv}_{f}^{o}\left(\boldsymbol{x}^{\prime \prime}\right) \wedge \text { Out }_{f}\left(\boldsymbol{x}^{\prime \prime}, \boldsymbol{x}^{\text {out }}\right)\right. \\
& \left.\quad \Longrightarrow \operatorname{Inv}_{f}^{o}(\boldsymbol{x}) \wedge \operatorname{Sum}_{f}^{o}\left(\boldsymbol{x}^{\text {in }}, \boldsymbol{x}^{\text {out }}\right)\right) \\
& \wedge\left(\operatorname{Inv}_{f}^{o}(\boldsymbol{x}) \wedge \operatorname{Trans}_{f}\left(\boldsymbol{x}, \boldsymbol{x}^{\prime}\right) \Longrightarrow \operatorname{Inv}_{f}^{o}\left(\boldsymbol{x}^{\prime}\right)\right)
\end{aligned}
$$

Lemma 2. Sum Su $_{f}^{o}$ and In $_{f}^{o}$ are over-approximating.

Proof sketch. By Lemma 1, CallCtx ${ }_{f}^{o}$ is over-approximating. Also, the summaries Sums $s_{f}^{o}$ are initially assumed to be true, i.e. over-approximating. Hence, given that $\mathrm{CallCtx}_{f}^{o}$ and Summs ${ }_{f}^{o}$ are over-approximating, Sum $_{f}^{o}$ and $\operatorname{Inv}_{f}^{o}$ are overapproximating by the soundness of the synthesis (Thm. 3).

Example 3. Let us consider procedure $h$ in Fig. 1. We have computed CallCtx $x_{h_{0}}^{o}\left((y),\left(r_{h}\right)\right)=\left(1 \leq y \leq M \wedge 0 \leq r_{h} \leq M\right)$ (with actual parameters renamed to formal ones). Then, we need to obtain witnesses Inv ${ }_{h_{0}}^{o}$ and Sum ${ }_{h_{0}}^{o}$ to the satifiability of the instantiation of Def. 5 (for procedure h) as given below.

$$
\begin{aligned}
& \exists \text { Inv }_{h_{0}}^{o}, \text { Sum }_{h_{0}}^{o}: \forall y, x, x^{\prime}, y^{\prime}, x^{\prime \prime}, y^{\prime \prime}, r_{f}: \\
& 1 \leq y \leq M \wedge 0 \leq r_{h} \leq M \wedge \text { true } \\
& \quad\left(( x = 0 \wedge y ^ { \prime } = y ) \wedge \operatorname { I n v } v _ { h } ^ { o } ( ( x ^ { \prime \prime } , y ^ { \prime \prime } ) ) \wedge \left(r_{h}=x^{\prime \prime} \wedge \neg\left(x^{\prime \prime}<10\right)\right.\right. \\
& \left.\quad \Longrightarrow \operatorname{Inv} o\left(\left(x, y^{\prime}\right)\right) \wedge \operatorname{Sum}_{h}^{o}\left((y),\left(r_{h}\right)\right)\right) \\
& \wedge\left(\operatorname{Inv}_{h}^{o}((x, y)) \wedge\left(x^{\prime}=(x+y \wedge x<10) \wedge y=y^{\prime}\right)\right. \\
& \left.\quad \Longrightarrow \operatorname{Inv}_{h}^{o}\left(\left(x^{\prime}, y^{\prime}\right)\right)\right) \\
& \text { A solution is Inv } \text { In }_{h_{0}}^{o}=(0 \leq x \leq M \wedge 1 \leq y \leq M) \text { and } \text { Sum }_{h_{0}}^{o}= \\
& \left(1 \leq y \leq M \wedge 10 \leq r_{h} \leq M\right), \text { for instance. }
\end{aligned}
$$

Remark 1. Since Def. 4 and Def. 5 are interdependent, we can compute them iteratively until a fixed point is reached in order to improve the precision of calling contexts, invariants and summaries. However, for efficiency reasons, we perform only the first iteration of this (greatest) fixed point computation.

Lemma 3 (compTermArg). A procedure $f$ with forward invariants Inv ${ }_{f}^{o}$ terminates if there is a termination argument $R R_{f}$ :

$$
\begin{aligned}
& \exists R R_{f}: \forall \boldsymbol{x}, \boldsymbol{x}^{\prime}: \\
& \quad \operatorname{Inv}_{f}^{o}(\boldsymbol{x}) \wedge \operatorname{Trans}_{f}\left(\boldsymbol{x}, \boldsymbol{x}^{\prime}\right) \wedge \operatorname{Summs}_{f}^{o} \wedge \text { Assertions }_{f}(\boldsymbol{x}) \\
& \quad \Longrightarrow R R_{f}\left(\boldsymbol{x}, \boldsymbol{x}^{\prime}\right)
\end{aligned}
$$

Assertions in this formula correspond to assert () statements in the code. They can be assumed to hold because assertion-violating traces terminate. Over-approximating forward information may lead to inclusion of spurious nonterminating traces. For that reason, we might not find a termination argument although the procedure is terminating. As we essentially under-approximate the set of terminating procedures, we will not give false positives. Regarding the solving algorithm for this formula, we refer to Sec. V.

Example 4. Let us consider function h in Fig. 1. Assume we have the invariant $0 \leq x \leq M \wedge 1 \leq y \leq M$. Thus, we have to solve

$$
\begin{gathered}
\exists R R_{h}: 0 \leq x \leq M \wedge 1 \leq y \leq M \wedge x^{\prime}=x+y \wedge x<10 \wedge y^{\prime}=y \wedge \\
\text { true } \wedge \text { true } \Longrightarrow R R_{h}\left((x, y),\left(x^{\prime}, y^{\prime}\right)\right)
\end{gathered}
$$

When using a linear ranking function template $c_{1} \cdot x+c_{2} \cdot y$, we obtain as solution, for example, $R R_{h}=\left(-x>-x^{\prime}\right)$.

If there is no trace from procedure entry to exit, then we can prove non-termination, even when using over-approximations:

Lemma 4 (line 7 of analyze). A procedure $f$ in forward calling context CallCtx ${ }_{f}^{o}$, and forward invariants Inv $_{f}^{o}$ never terminates if its summary Sum $_{f}^{o}$ is false.

Termination information is then propagated in the (acyclic) call graph (join in line 13 in Algorithm 2):

Proposition 1. A procedure $f$ is declared

(1) non-terminating if it is non-terminating by Lemma 4.

(2) terminating if

(a) all its procedure calls $h_{i}$ that are potentially reachable (i.e. with CallCtx $x_{h_{i}}^{o} \neq$ false) are declared terminating, and

(b) $f$ itself is terminating according to Lemma 3;

(3) potentially non-terminating, otherwise.

Our implementation is more efficient than Algorithm 2 because it avoids computing a termination argument for $f$ if one of its callees is potentially non-terminating.

Theorem 1. If the entry procedure of a program is declared terminating, then the program terminates universally. If the entry procedure of a program is declared non-terminating, then the program never terminates.

Proof sketch. By induction over the acyclic call graph using Prop. 1. 


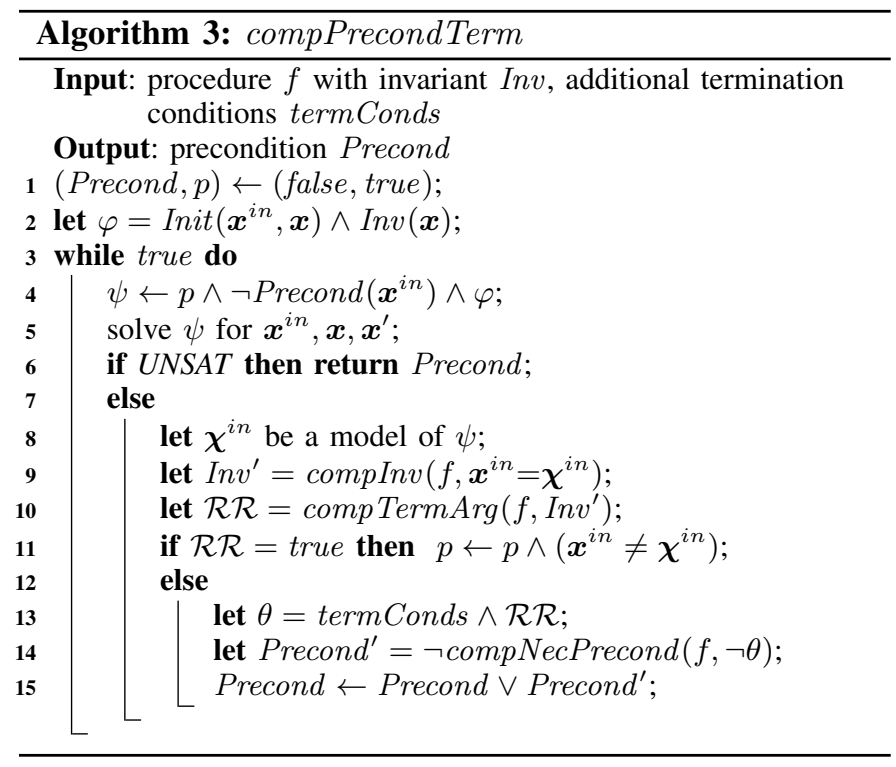

\section{B. Preconditions for Termination}

Before introducing conditional termination, we have to talk about preconditions for termination.

If a procedure terminates conditionally like procedure $h$ in Fig. 1 compTermArg (Lemma 3) will not be able to find a satisfying predicate $R R$. However, we would like to know under which preconditions, i.e. values of $y$ in above example, the procedure terminates.

We can state this problem as defined in Def. 3. In Algorithm 3 we search for Precond, Inv, and $R R$ in an interleaved manner. Note that false is a trivial solution for Precond; we thus have to aim at finding a good under-approximation of the maximal solution (weakest precondition) for Precond.

We bootstrap the process by assuming Precond $=$ false and search for values of $\boldsymbol{x}^{\text {in }}$ (Line 5). If such a value $\chi^{i n}$ exists, we can compute an invariant under the precondition candidate $\boldsymbol{x}^{i n}=\chi^{i n}$ (Line 9) and use Lemma 3 to search for the corresponding termination argument (Line 10).

If we fail to find a termination argument $(\mathcal{R} \mathcal{R}=$ true $)$, we block the precondition candidate (Line 11) and restart the bootstrapping process. Otherwise, the algorithm returns a termination argument $\mathcal{R} \mathcal{R}$ that is valid for the concrete value $\chi^{i n}$ of $\boldsymbol{x}^{i n}$. Now we need to find a sufficiently weak Precond for which $\mathcal{R} \mathcal{R}$ guarantees termination. To this end, we compute an over-approximating precondition for those inputs for which we cannot guarantee termination $(\neg \theta$ in Line 14 , which includes additional termination conditions coming from the backward calling context and preconditions of procedure calls, see Sec. IV-C). The negation of this precondition is an underapproximation of those inputs for which $f$ terminates. Finally, we add this negated precondition to our Precond (Line 15) before we start over the bootstrapping process to find precondition candidates outside the current precondition ( $\neg$ Precond) for which we might be able to guarantee termination.

Example 5. Let us consider again function h in Fig. 1. This time, we will assume we have the invariant $0 \leq x \leq M$ (with $\left.M:=2^{32}-1\right)$. We bootstrap by assuming Precond $=$ false and searching for values of $y$ satisfying true $\wedge \neg$ false $\wedge x=0 \wedge$ $0 \leq x \leq M$. One possibility is $y=0$. We then compute the invariant under the precondition $y=0$ and get $x=0$. Obviously, we cannot find a termination argument in this case. Hence, we start over and search for values of $y$ satisfying $y \neq 0 \wedge \neg$ false $\wedge x=0 \wedge 0 \leq x \leq M$. This formula is for instance satisfied by $y=1$. This time we get the invariant $0 \leq x \leq 10$ and the ranking function $-x$. Thus, we have to solve

$$
\begin{gathered}
\exists \boldsymbol{e}: \mathcal{P}(y, \boldsymbol{e}) \wedge 0 \leq x \leq M \wedge x^{\prime}=x+y \wedge x<10 \\
\Rightarrow \neg\left(-x>-x^{\prime}\right)
\end{gathered}
$$

to compute an over-approximating precondition over the template $\mathcal{P}$ (for details on templates see Section $V$ ). In this case, $\mathcal{P}(y, e)$ turns out to be $y=0$, therefore its negation $y \neq 0$ is the Precond that we get. Finally, we have to check for further precondition candidates, but $y \neq 0 \wedge \neg(y \neq$ 0) $\wedge x=0 \wedge 0 \leq x \leq M$ is obviously UNSAT. Hence, we return the sufficient precondition for termination $y \neq 0$.

\section{Conditional Termination}

We now extend the formalisation to Algorithm 1, which additionally requires the computation of under-approximating calling contexts and sufficient preconditions for termination (procedure compPrecondTerm, see Alg. 3).

First, compPrecondTerm computes in line 9 an overapproximating invariant $I n v_{f p}^{o}$ entailed by the candidate precondition. Inv $v_{f p}^{o}$ is computed through Def. 5 by conjoining the candidate precondition to the antecedent. Then, line 10 computes the corresponding termination argument $R R_{f}$ by applying Lemma 3 using $I n v_{f p}^{o}$ instead of $I n v_{f}^{o}$. Since the termination argument is under-approximating, we are sure that $f$ terminates for this candidate precondition if $R R_{f} \neq$ true.

Then, in line 14 of compPrecondTerm, we compute underapproximating (sufficient) preconditions for traces satisfying the termination argument $R R$ via over-approximating the traces violating $R R$.

Now, we are left to specify the formulae corresponding to the following functions:

- compCallCtx ${ }^{u}$ (Def. 6)

- compNecPrecond (Def. 7)

We use the superscript $\tilde{u}$ to indicate negations of underapproximating information. We compute under-approximating calling contexts as follows:

Definition 6 ( compCallCtx $\left.{ }^{u}\right)$. The backward calling context CallCtx $u_{h_{i}}^{u}$ for procedure call $h_{i}$ in procedure $f$ in backward calling context CallCtx $u_{f}^{u}$ and forward invariants Inv $_{f}^{o}$ is CallCtx $_{h_{i}}^{u} \equiv \neg$ CallCtx $_{h_{i}}^{\widetilde{u}}$, the negation of a satisfiability 
witnesses for:

$$
\begin{aligned}
& \exists \text { CallCtx }{ }_{h_{i}}^{\tilde{u}}, \operatorname{Inv}{ }_{f}^{\tilde{u}}: \forall \boldsymbol{x}^{i n}, \boldsymbol{x}, \boldsymbol{x}^{\prime}, \boldsymbol{x}^{p_{-} i n}{ }_{i}, \boldsymbol{x}^{p_{-} \text {out }}{ }_{i}, \boldsymbol{x}^{\text {out }} \text { : } \\
& \neg \operatorname{CallCtx}_{f}^{u}\left(\boldsymbol{x}^{i n}, \boldsymbol{x}^{\text {out }}\right) \wedge \operatorname{Inv}_{f}^{o}(\boldsymbol{x}) \wedge \\
& \text { Summs }_{f}^{o} \wedge \text { Summs }_{f}^{\tilde{u}} \wedge \text { Assertions }_{f}(\boldsymbol{x}) \Longrightarrow \\
& \left(\operatorname{Out}\left(\boldsymbol{x}, \boldsymbol{x}^{\text {out }}\right) \Longrightarrow \operatorname{Inv}_{f}^{\tilde{u}}(\boldsymbol{x})\right) \\
& \wedge\left(\operatorname{Inv}_{f}^{\tilde{u}}\left(\boldsymbol{x}^{\prime}\right) \wedge \operatorname{Trans}\left(\boldsymbol{x}, \boldsymbol{x}^{\prime}\right)\right. \\
& \left.\Longrightarrow \operatorname{Inv}_{f}^{\tilde{u}}(\boldsymbol{x}) \wedge\left(g_{h_{i}} \Longrightarrow \operatorname{CallCtx}_{h_{i}}^{\tilde{u}}\left(\boldsymbol{x}^{p_{-} \text {in }}, \boldsymbol{x}^{p_{-} \text {out }}\right)\right)\right)
\end{aligned}
$$

with Summs Su $_{f}^{\tilde{u}}=\bigwedge_{\text {calls } h_{j} \text { in } f} \quad g_{h_{j}} \Longrightarrow$

$$
\neg S u{ }^{u}[h]\left(\boldsymbol{x}^{p_{-} i n}{ }_{j}, \boldsymbol{x}^{p_{-} \text {out }}{ }_{j}\right)
$$

Lemma 5. CallCtx ${ }_{h_{i}}^{u}$ is under-approximating.

Proof sketch. The computation is based on the negation of the under-approximating calling context of $f$ and the negated under-approximating summaries for the function calls in $f$. By Thm. 3, this leads to an over-approximation of the negation of the calling context for $h_{i}$.

Example 6. Let us assume that in procedure $f$ in Fig. 1, we have CallCtx $f_{f}^{u}\left((z),\left(r_{f}\right)\right)=\left(11 \leq r_{f} \leq M\right)$, i.e. $f$ is called in a context where a return value of less than 11 would cause non-termination of the caller. Then, we instantiate Def. 6 (for procedure f) to compute CallCtx $\mathrm{h}_{0}^{u}$. We assume that we have already computed the over-approximating summary Sum $_{h_{0}}^{o}=\left(1 \leq z \leq M \wedge 10 \leq w_{1} \leq M\right)$, but not yet computed an under-approximating summary for $h$, thus, Sum $_{h}^{u}$ is false.

$$
\begin{aligned}
& \exists \text { CallCtx }_{h_{0}}^{\tilde{u}}, \text { Inv }_{f}^{\tilde{u}}: \forall z, w_{1}, w, w^{\prime}, z^{\prime}, g, g^{\prime}, r_{f}: \\
& 0 \leq r_{f} \leq 10 \wedge \text { true } \wedge \\
& \left(z>0 \Rightarrow 1 \leq z \leq M \wedge 10 \leq w_{1} \leq M\right) \wedge(z>0 \Rightarrow \text { true }) \wedge \text { true } \Longrightarrow \\
& \quad\left(r_{f}=w \wedge \neg g \Longrightarrow \operatorname{Inv}_{f}^{\tilde{u}}((w, z, g))\right) \\
& \wedge\left(\operatorname{Inv}_{f}^{\tilde{u}}((w, z, g)) \wedge\right. \\
& \quad g \wedge h_{0}\left((z),\left(w_{1}\right)\right) \wedge w^{\prime}=\left(z>0 ? w_{1}: w\right) \wedge z^{\prime}=z \wedge \neg g^{\prime} \\
& \quad \Longrightarrow \operatorname{Inv}_{f}^{\tilde{u}}\left(\left(w^{\prime}, z^{\prime}, g^{\prime}\right)\right) \wedge\left(z>0 \Rightarrow \operatorname{CallCtx}_{h_{i}}^{\tilde{u}}\left((z),\left(w_{1}\right)\right)\right)
\end{aligned}
$$

A solution is Inv $_{f}^{\tilde{u}}=$ true, and CallCtx $\tilde{h}_{h_{0}}^{\tilde{u}}=(1 \leq z \leq M \wedge$ $\left.0 \leq w_{1} \leq 10\right)$, i.e. CallCtx $h_{h_{0}}^{u}=\left(z=0 \vee 11 \leq w_{1} \leq M\right)$.

Definition 7 (Line 14 of compPrecondTerm). A precondition for termination Precond $u_{f}^{u}$ in backward calling context CallCtx $u_{f}^{u}$ and with forward invariants Inv ${ }_{f}^{o}$ is Precond ${ }_{f}^{u} \equiv$ $\neg$ Precond $d_{f}^{\tilde{u}}$, i.e. the negation of a satisfiability witness Precond $\tilde{u}_{f}^{\tilde{u}}$ for:

$$
\begin{aligned}
& \exists \operatorname{Precond}_{h_{i}}^{\tilde{u}}, \operatorname{Inv}_{f}^{\tilde{u}}, \operatorname{Sum}_{f}^{\tilde{u}}: \forall \boldsymbol{x}^{i n}, \boldsymbol{x}, \boldsymbol{x}^{\prime}, \boldsymbol{x}^{\prime \prime}, \boldsymbol{x}^{\text {out }} \text { : } \\
& \neg \operatorname{CallCtx}_{f}^{u}\left(\boldsymbol{x}^{\text {in }}, \boldsymbol{x}^{\text {out }}\right) \wedge \operatorname{Inv}_{f}^{o}(\boldsymbol{x}) \wedge \\
& \text { Summs }_{f}^{o} \wedge \text { Summs }_{f}^{\tilde{u}} \wedge \text { Assertions }_{f}(\boldsymbol{x}) \Longrightarrow \\
& \left(\operatorname{Init}\left(\boldsymbol{x}^{i n}, \boldsymbol{x}^{\prime \prime}\right) \wedge \operatorname{Inv}{ }_{f}^{\tilde{u}}\left(\boldsymbol{x}^{\prime \prime}\right) \wedge \operatorname{Out}\left(\boldsymbol{x}, \boldsymbol{x}^{\text {out }}\right)\right. \\
& \left.\Longrightarrow \operatorname{Inv}_{f}^{\tilde{u}}(\boldsymbol{x}) \wedge \operatorname{Sum}_{f}^{\tilde{u}}\left(\boldsymbol{x}^{i n}, \boldsymbol{x}^{\text {out }}\right) \wedge \operatorname{Precond}_{f}^{\tilde{u}}\left(\boldsymbol{x}^{i n}\right)\right) \\
& \wedge\left(\left(\neg \mathcal{R}_{f}\left(\boldsymbol{x}, \boldsymbol{x}^{\prime}\right) \vee \text { Preconds }_{f}^{\tilde{u}} \vee \neg \text { CallCtx }_{f}^{u}\left(\boldsymbol{x}^{\text {in }}, \boldsymbol{x}^{\text {out }}\right) \wedge\right.\right. \\
& \left.\operatorname{Init}\left(\boldsymbol{x}^{\text {in }}, \boldsymbol{x}\right) \wedge \operatorname{Out}\left(\boldsymbol{x}^{\prime}, \boldsymbol{x}^{\text {out }}\right)\right) \wedge \\
& \left.\operatorname{Inv} v_{f}^{\tilde{u}}\left(\boldsymbol{x}^{\prime}\right) \wedge \operatorname{Trans}\left(\boldsymbol{x}, \boldsymbol{x}^{\prime}\right) \Longrightarrow \operatorname{Inv}_{f}^{\tilde{u}}(\boldsymbol{x})\right)
\end{aligned}
$$

with Preconds $\tilde{u}_{f}^{\tilde{u}}=\bigvee_{\text {calls } h_{j} \text { in } f} g_{h_{j}} \Longrightarrow$

$$
\neg \text { Precond }^{u}[h]\left(\boldsymbol{x}^{p_{-} i n}{ }_{j}, \boldsymbol{x}^{p_{-} \text {out }}{ }_{j}\right) \text {. }
$$

This formula is similar to Def. 5, but w.r.t. backward calling contexts and summaries, and strengthened by the (forward) invariants $I n v_{f}^{o}$. We denote the negation of the witnesses found for the summary and the invariant by $\operatorname{Sum}_{f}^{u} \equiv \neg S u m_{f}^{\tilde{u}}$ and $\operatorname{In} v_{f}^{u} \equiv \neg \operatorname{In} v_{f}^{\tilde{u}}$, respectively.

Lemma 6. Precond $d_{f}^{u}$, Sum Su $_{f}^{u}$ Inv ${ }_{f}^{u}$ are underapproximating.

Proof sketch. We compute an over-approximation of the negation of the precondition w.r.t. the negation of the under-approximating termination argument and the negation of further under-approximating information (backward calling context, preconditions of procedure calls) — by the soundness of the synthesis (see Thm. 3 in Sec. V), this over-approximates the non-terminating traces, and hence under-approximates the terminating ones. Hence, the precondition is a sufficient precondition for termination. The term $\neg R R_{f}\left(\boldsymbol{x}, \boldsymbol{x}^{\prime}\right) \vee$ Preconds $\tilde{u}_{f}^{\widetilde{u}} \vee \neg \operatorname{CallCtx}_{f}^{u}\left(\boldsymbol{x}^{\text {in }}, \boldsymbol{x}^{\text {out }}\right) \wedge$ $\operatorname{Init}\left(\boldsymbol{x}^{\text {in }}, \boldsymbol{x}\right) \wedge \operatorname{Out}\left(\boldsymbol{x}^{\prime}, \boldsymbol{x}^{\text {out }}\right)$ characterises non-terminating states in the invariants of $f$ : for these, either the termination argument for $f$ is not satisfied or the precondition for termination of one of the callees does not hold or we are outside the calling context.

Example 7. We will instantiate Def. 7 for procedure $h$ in Fig. 1, assuming that we have CallCtx ${ }_{h}^{u}\left((y),\left(r_{h}\right)\right)=$ $\left(y=0 \vee 11 \leq r_{h} \leq M\right)$, as computed in the previous example. Inv $v_{h}^{o}=$ true and $h$ does not have any procedure calls, thus Summs $s_{h}^{o}=$ true, Summs $s_{h}^{\tilde{u}}=$ true, and Preconds $s_{h}^{\tilde{u}}=$ false. Assume we have the termination argument candidate $R R_{h}=$ $\left(-x>-x^{\prime}\right)$.

The second conjunct in the consequent of the top-level implication is satisfied either for $y=0$ making $\neg R R_{h}$ true or $y \leq 10$ violating the calling context (third disjunct).

Hence, a solution is Precond $\tilde{u}_{h}=(0 \leq y \leq 10)$, Inv $v_{h}^{\tilde{u}}=(0 \leq x \leq 19 \wedge 0 \leq y \leq 10)$, and Sum $_{h}^{\tilde{u}}=(0 \leq y \leq 10 \wedge$ $\left.0 \leq r_{h} \leq 10\right)$. I.e. a sufficient precondition for termination is Precond $_{h}^{u}=(11 \leq y \leq M)$.

Theorem 2. A procedure $f$ terminates for all values of $\boldsymbol{x}^{i n}$ satisfying Precond ${ }_{f}^{u}$.

Proof sketch. By induction over the acyclic call graph using Lemmae 5 and 6.

\section{Context-Sensitive Summaries}

The key idea of interprocedural analysis is to avoid reanalysing procedures that are called multiple times. For that reason, Algorithm 1 first checks whether it can re-use already computed information. For that purpose, summaries are stored as implications CallCtx $^{o} \Rightarrow$ Sum $^{\circ}$. As the call graph is traversed, the possible calling contexts CallCtx $_{h_{i}}^{o}$ for a procedure $h$ are collected over the call sites $i$. NeedToReAnalyze ${ }^{o}$ (Line 5 in Alg. 1) checks whether the current calling context 
CallCtx $x_{h_{i}}^{o}$ is subsumed by calling contexts $\bigvee_{i}$ CallCtx $_{h_{i}}^{o}$ that we have already encountered, and if so, $\operatorname{Sums}^{o}[h]$ is reused; otherwise it needs to be recomputed and joined conjunctively with previously inferred summaries. The same considerations apply to invariants, termination arguments and preconditions.

\section{Template-Based Static Analysis}

In this section, we give a brief overview of our synthesis engine, which serves as a backend for our approach (it solves the formulae in Definitions 4, 5, 7, and 6 (see Sec. IV)).

Our synthesis engine employs template-based static analysis to compute ranking functions, invariants, summaries, and calling contexts, i.e., implementations of functions compInvSum ${ }^{\circ}$ and compCallCtx ${ }^{\circ}$ from the second-order constraints defined in Sec. IV. To be able to effectively solve second-order problems, we reduce them to first-order by restricting the space of solutions to expressions of the form $\mathcal{T}(\boldsymbol{x}, \boldsymbol{d})$ where

- $\boldsymbol{d}$ are parameters to be instantiated with concrete values and $\boldsymbol{x}$ are the program variables.

- $\mathcal{T}$ is a template that gives a blueprint for the shape of the formulas to be computed. Choosing a template is analogous to choosing an abstract domain in abstract interpretation. To allow for a flexible choice, we consider template polyhedra [9].

We state here a soundness result:

Theorem 3. Any satisfiability witness $\boldsymbol{d}$ of the reduction of the second order constraint for invariants in Def. 1 using template $\mathcal{T}$

$$
\begin{aligned}
\exists \boldsymbol{d}, \forall \boldsymbol{x}^{i n}, \boldsymbol{x}, \boldsymbol{x}^{\prime}: & \operatorname{Init}\left(\boldsymbol{x}^{i n}, \boldsymbol{x}\right) \Longrightarrow \mathcal{T}(\boldsymbol{x}, \boldsymbol{d}) \\
\wedge & \mathcal{T}(\boldsymbol{x}, \boldsymbol{d}) \wedge \operatorname{Trans}\left(\boldsymbol{x}, \boldsymbol{x}^{\prime}\right) \Longrightarrow \mathcal{T}\left(\boldsymbol{x}^{\prime}, \boldsymbol{d}\right)
\end{aligned}
$$

satisfies $\forall \boldsymbol{x}: \operatorname{Inv}(\boldsymbol{x}) \Longrightarrow \mathcal{T}(\boldsymbol{x}, \boldsymbol{d})$, i.e. $\mathcal{T}(\boldsymbol{x}, \boldsymbol{d})$ is a sound over-approximating invariant. Similar soundness results hold true for summaries and calling contexts.

This ultimately follows from the soundness of abstract interpretation [10]. Similar approaches have been described, for instance, by [11], [12], [13]. However, these methods consider programs over mathematical integers.

Ranking functions require specialised synthesis techniques. To achieve both expressiveness and efficiency, we generate linear lexicographic functions [14], [15]. Our ranking-function synthesis approach is similar to the TAN tool [16] but extends the approach from monolithic to lexicographic ranking functions. Further, unlike TAN, our synthesis engine is much more versatile and configurable, e.g., it also produces summaries and invariants.

Due to space limitations, we refer to the extended version [7], which includes a detailed description of the synthesis engine, our program encoding, encoding of bit-precise arithmetic, and tailored second-order solving techniques for the different constraints that occur in our analysis. In the following section, we discuss the implementation.

\section{IMPLEMENTATION}

We have implemented the algorithm in 2LS [17], a static analysis tool for C programs built on the CPROVER framework, using MiniSat 2.2.0 as back-end solver. Other SAT and SMT solvers with incremental solving support would also be applicable. Our approach enables us to use a single solver instance per procedure to solve a series of second-order queries as required by Alg. 1. This is essential as our synthesis algorithms make thousands of solver calls. Architectural settings (e.g. bitwidths) can be provided on the command line.

Discussions about technical issues w.r.t. bit-preciseness and the computation of intraprocedural termination arguments can be found in the extended version [7].

\section{EXPERIMENTS}

We performed experiments to support the following claims:

1) Interprocedural termination analysis (IPTA) is faster than monolithic termination analysis (MTA).

2) The precision of IPTA is comparable to MTA.

3) $2 \mathrm{LS}$ outperforms existing termination analysis tools.

4) 2LS's analysis is bit-precise.

5) 2LS computes usable preconditions for termination.

We used the product line benchmarks of the [18] benchmark repository. In contrast to other categories, this benchmark set contains programs with non-trivial procedural structure. This benchmark set contains 597 programs with 1100 to 5700 lines of code (2705 on average), ${ }^{4} 33$ to 136 procedures (67 on average), and 4 to 10 loops (5.5 on average). Of these benchmarks, 264 terminate universally, whereas 333 never terminate.

The experiments were run on a Xeon X5667 at $3 \mathrm{GHz}$ running Fedora 20 with 64-bit binaries. Memory and CPU time were restricted to $16 \mathrm{~GB}$ and 1800 seconds per benchmark, respectively (using [19]). Using 2LS with interval templates was sufficient to obtain reasonable precision.

Modular termination analysis is fast We compared IPTA with MTA (all procedures inlined). Table I shows that IPTA times out on $2.3 \%$ of the benchmarks vs. $39.7 \%$ for MTA. The geometric mean speed-up of IPTA w.r.t. MTA on the benchmarks correctly solved by both approaches is 1.37 .

In order to investigate how the $30 \mathrm{~m}$ timeout affects MTA, we randomly selected 10 benchmarks that timed out for $30 \mathrm{~m}$ and re-ran them: 1 finished in $32 \mathrm{~m}, 3$ after more than $1 \mathrm{~h}$, 6 did not finish within $2 \mathrm{~h}$.

Modular termination analysis is precise Again, we compare IPTA with MTA. Table I shows that IPTA proves $94 \%$ of the terminating benchmarks, whereas only $10 \%$ were proven by MTA. MTA can prove all never-terminating benchmarks including 13 benchmarks where IPTA times out. MTA times out on the benchmarks that cause 13 additional potentially non-terminating outcomes for IPTA.

2LS outperforms existing termination analysis tools We compared 2LS with two termination tools for $\mathrm{C}$ programs from

\footnotetext{
${ }^{4}$ Measured using cloc 1.53 .
} 
TABLE I

TOOL COMPARISON (* see text).

\begin{tabular}{|c|c|c|c|c|}
\hline & 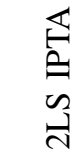 & 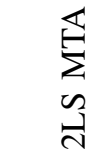 & 芯 & $\begin{array}{l}\stackrel{\Xi}{\Xi} \\
. \Xi \\
. \Xi\end{array}$ \\
\hline terminating & 249 & 26 & 18 & 50 \\
\hline non-terminating & 320 & 333 & 3 & $324^{*}$ \\
\hline potentially non-term. & 14 & 1 & 425 & 0 \\
\hline timed out & 14 & 237 & 150 & 43 \\
\hline errors & 0 & 0 & 1 & 180 \\
\hline total run time $(\mathrm{h})$ & 58.7 & 119.6 & 92.8 & 23.9 \\
\hline
\end{tabular}

the SV-COMP termination competition, namely TAN [20] and Ultimate [21].

Unfortunately, the tools [22], [23], [24], [25], and [26] have limitations regarding the subset of $\mathrm{C}$ that they can handle that make them unable to analyze any of the benchmarks out of the box. We describe these limitations in the experiments log in [17]. Unfortunately, we did not succeed to generate the correct input files in the intermediate formats required by T2 [27] and KiTTeL [28] using the recommended frontends [29] and [30].

TAN [16] and KiTTeL/KoAT [5] support bit-precise C semantics. Ultimate uses mathematical integer reasoning but tries to ensure conformance with bit-vector semantics. Also, Ultimate uses a semantic decomposition of the program [31] to make its analysis efficient.

For each of the tools, Table I lists the number of instances solved, timed out or aborted because of an internal error. We also give the total run time, which shows that analysis times are roughly halved by the modular/interprocedural approaches (2LS IPTA, Ultimate) in comparison with the monolithic approaches (2LS MTA, TAN). Ultimate spends less time on those benchmarks that it can prove terminating, however, these are only $19 \%$ of the terminating benchmarks (vs. $94 \%$ for 2LS). If Ultimate could solve those 180 benchmarks on which it fails due to unsupported features of $\mathrm{C}$, we would expect its performance to be comparable to 2LS.

Ultimate and 2LS have different capabilities regarding nontermination. 2LS can show that a program never terminates for all inputs, whereas Ultimate can show that there exists a potentially non-terminating execution. To make the comparison fair, we counted benchmarks flagged as potentially non-terminating by Ultimate, but which are actually never-terminating, in the non-terminating category in Table I (marked *).

2LS's analysis is bit-precise We compared 2LS with Loopus on a collection of 15 benchmarks (ABC_ex01.C to ABC_ex15.c) taken from the Loopus benchmark suite [23]. While they are short (between 7 and 41 LOC), the main characteristic of these programs is the fact that they exhibit different terminating behaviours for mathematical integers and bit-vectors. For illustration, ABC_ex15.c (Fig. 3) terminates with mathematical integers, but not with machine integers if, for instance, m equals INT_MAX. Next, we summarise

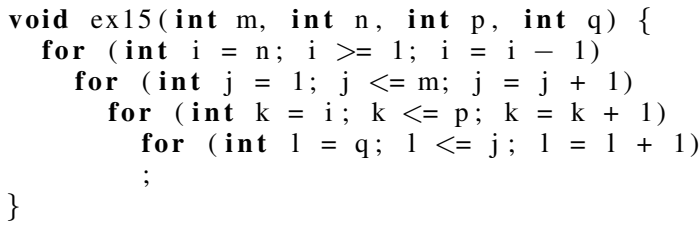

Fig. 3. Example ABC_ex15.c from the Loopus benchmarks.

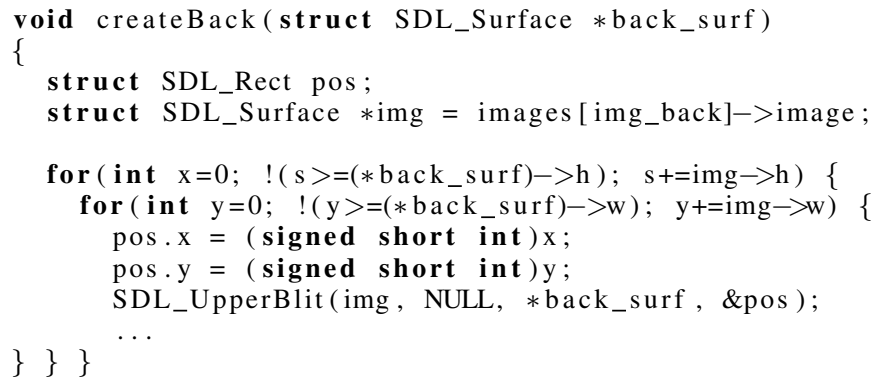

Fig. 4. Example createBack from Debian package abe.

the results of our experiments on these benchmarks when considering machine integers:

- Only 2 of the programs terminate, and are correctly identified by both $2 \mathrm{LS}$ and Loopus.

- For the rest of 13 non-terminating programs, Loopus claims they terminate, whereas 2LS correctly classifies 9 as potentially non-terminating (including ABC_ex15.C in Fig. 3) and times out on 4.

2LS computes usable preconditions for termination This experiment was performed on benchmarks extracted from Debian packages and the linear algebra library CLapack.

The quality of preconditions, i.e. usability or ability to help the developer to spot problems in the code, is difficult to quantify. We give several examples where functions terminate conditionally. The abe package of Debian contains a function, given as Fig. 4, where increments of the iteration in a loop are not constant but dynamically depend on the dimensions of an image data structure. Here, 2LS infers the precondition $i m g \rightarrow h>0 \wedge i m g \rightarrow w>0$.

The example in Fig. 5 is taken from the benchmark basename in the busybox-category of SVCOMP 2015, which contains simplified versions of Debian packages. The termination of function full_write depends on the return value of its callee function safe_write. Here, 2LS infers the calling context $c c>0$, i.e. the contract for the function safe_write, such that the termination of full_write is guaranteed. Given a proof that safe_write terminates and returns a strictly positive value regardless of the arguments it is called with, we can conclude that full_write terminates universally.

The program in Fig. 6 is a code snippet taken from the summation procedure sasum within [32], the $C$ version of the popular LAPACK linear algebra library. The loop in procedure $\mathrm{f}$ does not terminate if $i n c x=0$. If incx $>0($ inc $x<0)$ the termination argument is that $i$ increases (decreases). Therefore, inc $x \neq 0$ is a termination precondition for $\mathrm{f}$. 


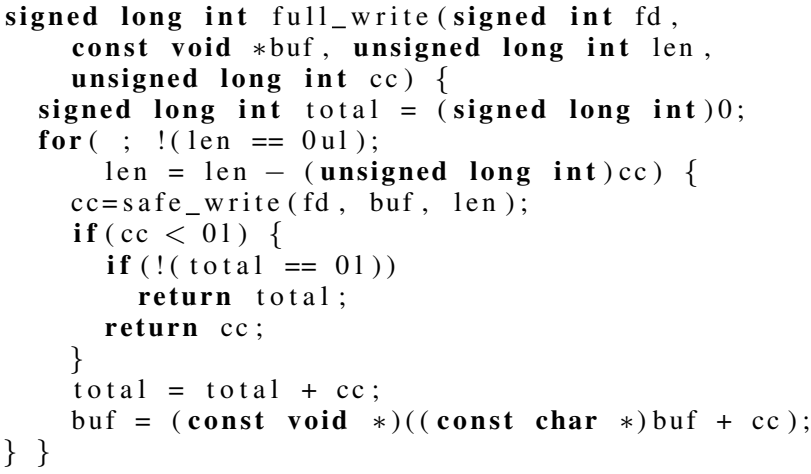

Fig. 5. Example from SVCOMP 2015 busybox.

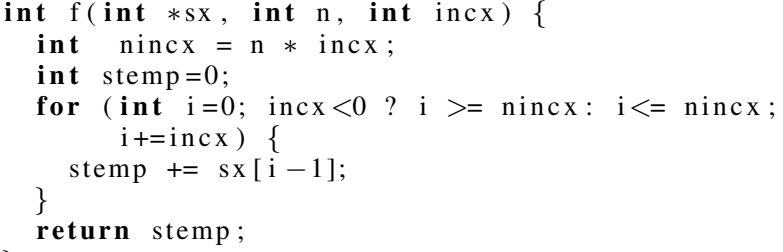

Fig. 6. Non-unit increment from CLapack.

\section{LIMITATIONS, RELATED WORKS AND FUTURE DIRECTIONS}

Our approach makes significant progress towards analysing real-world software, advancing the state-of-the-art of termination analysis of large programs. Conceptually, we decompose the analysis into a sequence of well-defined second-order predicate logic formulae with existentially quantified predicates. In addition to [33], we consider context-sensitive analysis, underapproximate backwards analysis, and make the interaction with termination analysis explicit. Notably, these seemingly tedious formulae are actually solved by our generic templatebased synthesis algorithm, making it an efficient alternative to predicate abstraction.

An important aspect of our analysis is that it is bit-precise. As opposed to the synthesis of termination arguments for linear programs over integers (rationals) [34], [35], [2], [36], [37], [14], [15], this subclass of termination analyses is substantially less covered. While [16], [38] present methods based on a reduction to Presburger arithmetic, and a template-matching approach for predefined classes of ranking functions based on reduction to SAT- and QBF-solving, [39] only compute intraprocedural termination arguments.

There are still a number of limitations to be addressed, all of which connect to open challenges subject to active research. While some are orthogonal (e.g., data structures, strings, refinement) to our interprocedural analysis framework, others (recursion, necessary preconditions) require extensions of it. In this section, we discuss related work, as well as, characteristics and limitations of our analysis, and future directions (cost analysis and concurrency).

Dynamically allocated data structures We currently ignore heap-allocated data. This limitation could be lifted by using specific abstract domains. For illustration, let us consider the following example traversing a singly-linked list.

List $\mathrm{x}$; while ( $\mathrm{x} !=$ NULL) $\{\mathrm{x}=\mathrm{x} \rightarrow$-next; $\}$

Deciding the termination of such a program requires knowledge about the shape of the data structure pointed by $x$, namely, the program only terminates if the list is acyclic. Thus, we would require an abstract domain capable of capturing such a property and also relate the shape of the data structure to its length. Similar to [15], we could use [40] in order to abstract heap-manipulating programs to arithmetic ones. Another option is using an abstract interpretation based on separation logic formulae which tracks the depths of pieces of heaps similarly to [41].

Strings and arrays Similar to dynamic data structures, handling strings and arrays requires specific abstract domains. String abstractions that reduce null-terminated strings to integers (indices, length, and size) are usually sufficient in many practical cases; scenarios where termination is dependent on the content of arrays are much harder and would require quantified invariants [42]. Note that it is favorable to run a safety checker before the termination checker. The latter can assume that assertions for buffer overflow checks hold which strengthens invariants and makes termination proofs easier.

Recursion We currently use downward fixed point iterations for computing calling contexts and invariants that involve summaries (see Remark 1). This is cheap but gives only imprecise results in the presence of recursion, which would impair the termination analysis. We could handle recursions by detecting cycles in the call graph and switching to an upward iteration scheme in such situations. Moreover, an adaptation regarding the generation of the ranking function templates is necessary. An alternative approach would be to make use of the theoretic framework presented in [43] for verifying total correctness and liveness properties of while programs with recursion.

Template refinement We currently use interval templates together with heuristics for selecting the variables that should be taken into consideration. This is often sufficient in practice, but it does not exploit the full power of the machinery in place. While counterexample-guided abstraction refinement (CEGAR) techniques are prevalent in predicate abstraction [44], attempts to use them in abstract interpretation are rare [45]. We consider our template-based abstract interpretation that automatically synthesises abstract transformers more amenable to refinement techniques than classical abstract interpretations where abstract transformers are implemented manually.

Sufficient preconditions to termination Conditional termination has recently attracted increased interest [8], [46], [47], [48], [49]. In this paper, we compute sufficient preconditions, i.e. under-approximating preconditions to termination via computing over-approximating preconditions to potential non-termination. The same concept is used by other works [8], [46]. However, they consider only a single procedure and do not leverage their results to perform interprocedural 
analysis on large benchmarks which adds, in particular, the additional challenge of propagating under-approximating information up to the entry procedure (e.g. [50]). Moreover, by contrast to Cook et al [8] who use an heuristic FINITEoperator left unspecified for bootstrapping their preconditions, our bootstrapping is systematic through constraint solving.

We could compute necessary preconditions by computing over-approximating preconditions to potential termination (and negating the result). However, this requires a method for proving that there exist non-terminating executions, which is a well-explored topic. While [51] dynamically enumerate lasso-shaped candidate paths for counterexamples, and then statically prove their feasibility, [52] prove nontermination via reduction to safety proving and [53] uses bi-abduction to construct summaries of terminating and non-terminating behaviors for each method. In order to prove both termination and non-termination, [54] compose several program analyses (termination provers for multi-path loops, non-termination provers for cycles, and safety provers).

Cost analysis A potential future application for our work is cost and resource analysis. Instances of this type of analyses are the worst case execution time (WCET) analysis [55], as well as bound and amortised complexity analysis [56], [57], [58]. The control flow refinement approach [59], [60] instruments a program with counters and uses progress invariants to compute worst case or average case bounds.

Concurrency Our current analysis handles single-threaded $\mathrm{C}$ programs. One way of extending the analysis to multithreaded programs is using the rely-guarantee technique which is proposed in [61], and explored in several works [62], [63], [64] for termination analysis. In our setting, the predicates for environment assumptions can be used in a similar way as invariants and summaries are used in the analysis of sequential programs.

\section{COnclusions}

While many termination provers mainly target small, hard programs, the termination analysis of larger code bases has received little attention. We present an algorithm for interprocedural termination analysis for non-recursive programs. To our knowledge, this is the first paper that describes in full detail the entire machinery necessary to perform such an analysis. Our approach relies on a bit-precise static analysis combining SMT solving, template polyhedra and lexicographic, linear ranking function templates. We provide an implementation of the approach in the static analysis tool 2LS, and demonstrate the applicability of the approach to programs with thousands of lines of code.

\section{REFERENCES}

[1] http://cve.mitre.org/cgi-bin/cvename.cgi?name=CVE-2009-1890.

[2] A. M. Ben-Amram and S. Genaim, "On the linear ranking problem for integer linear-constraint loops," in Principles of Programming Languages, pp. 51-62, ACM, 2013.

[3] J. Leike and M. Heizmann, "Ranking templates for linear loops," in Tools and Algorithms for the Construction and Analysis of Systems, vol. 8413 of LNCS, pp. 172-186, Springer, 2014.
[4] A. R. Bradley, Z. Manna, and H. B. Sipma, "Termination of polynomial programs," in Verification, Model Checking, and Abstract Interpretation, vol. 3385 of $L N C S$, pp. 113-129, Springer, 2005.

[5] S. Falke, D. Kapur, and C. Sinz, "Termination analysis of imperative programs using bitvector arithmetic," in Verified Software: Theories, Tools, Experiments, vol. 7152 of LNCS, pp. 261-277, Springer, 2012.

[6] D. Beyer, "Status report on software verification - (competition summary SV-COMP 2014)," in Tools and Algorithms for the Construction and Analysis of Systems, vol. 8413 of LNCS, Springer, 2014.

[7] H.-Y. Chen, C. David, D. Kroening, P. Schrammel, and B. Wachter, "Synthesising interprocedural bit-precise termination proofs (extended version)," tech. rep., University of Oxford, 2015. http://arxiv.org/abs/ 1505.04581.

[8] B. Cook, S. Gulwani, T. Lev-Ami, A. Rybalchenko, and M. Sagiv, "Proving conditional termination," in Computer-Aided Verification, vol. 5123 of LNCS, pp. 328-340, Springer, 2008.

[9] S. Sankaranarayanan, H. B. Sipma, and Z. Manna, "Scalable analysis of linear systems using mathematical programming," in Verification, Model Checking, and Abstract Interpretation, vol. 3385 of LNCS, pp. 25-41, Springer, 2005.

[10] P. Cousot and R. Cousot, "Abstract interpretation: A unified lattice model for static analysis of programs by construction or approximation of fixpoints," in Principles of Programming Languages, pp. 238-252, 1977.

[11] T. M. Gawlitza and H. Seidl, "Precise relational invariants through strategy iteration," in Computer Science Logic, vol. 4646 of LNCS, pp. 23-40, Springer, 2007.

[12] S. Gulwani, S. Srivastava, and R. Venkatesan, "Program analysis as constraint solving," in Programming Language Design and Implementation, pp. 281-292, ACM, 2008.

[13] Y. Li, A. Albarghouthi, Z. Kincaid, A. Gurfinkel, and M. Chechik, "Symbolic optimization with SMT solvers," in Principles of Programming Languages, pp. 607-618, ACM, 2014.

[14] A. R. Bradley, Z. Manna, and H. B. Sipma, "Linear ranking with reachability," in Computer-Aided Verification, pp. 491-504, 2005.

[15] B. Cook, A. See, and F. Zuleger, "Ramsey vs. lexicographic termination proving," in Tools and Algorithms for the Construction and Analysis of Systems, pp. 47-61, 2013.

[16] D. Kroening, N. Sharygina, A. Tsitovich, and C. M. Wintersteiger, "Termination analysis with compositional transition invariants," in ComputerAided Verification, vol. 6174 of LNCS, pp. 89-103, Springer, 2010.

[17] http://www.cprover.org/wiki/doku.php?id=2ls_for_program_analysis (version 0.1).

[18] https://svn.sosy-lab.org/software/sv-benchmarks/tags/svcomp14/ product-lines/.

[19] O. Roussel, "Controlling a solver execution with the runsolver tool," Journal on Satisfiability, Boolean Modeling and Computation, vol. 7, no. 4, pp. 139-144, 2011.

[20] http://www.cprover.org/termination/ (version SV-COMP-2014).

[21] M. Heizmann, D. Dietsch, J. Leike, B. Musa, and A. Podelski, "Ultimate automizer with array interpolation - (competition contribution)," in Tools and Algorithms for the Construction and Analysis of Systems, vol. 9035 of LNCS, Springer, 2015. http://ultimate.informatik.uni-freiburg.de/ (version SV-COMP-2015).

[22] T. Ströder, C. Aschermann, F. Frohn, J. Hensel, and J. Giesl, "AProVE: Termination and Memory Safety of C programs - (competition contribution)," in Tools and Algorithms for the Construction and Analysis of Systems, vol. 9035 of LNCS, Springer, 2015. http://aprove.informatik. rwth-aachen.de (version 2014).

[23] http://forsyte.at/software/loopus/ with http://sourceforge.net/projects/ virtualboximage/files/Ubuntu\%20Linux/11.10/ubuntu_11.10-x86.7z/ download.

[24] http://www.di.ens.fr/ urban/sv-comp2015.zip (version SV-COMP2015).

[25] T. C. Le, C. Gherghina, A. Hobor, and W. Chin, "A resource-based logic for termination and non-termination proofs," in ICFEM, vol. 8829 of LNCS, Springer, 2014. http://loris-7.ddns.comp.nus.edu.sg/ project/ hiptnt/plus/.

[26] A. Podelski and A. Rybalchenko, "ARMC: the logical choice for software model checking with abstraction refinement," in Practical Aspects of Declarative Languages, vol. 4354 of LNCS, Springer, 2007. https://www7.in.tum.de/ rybal/armc/ (version August 2011).

[27] http://research.microsoft.com/en-us/projects/t2/ (version 2014-10).

[28] https://github.com/s-falke/kittel-koat (revision c05eab4b3c).

[29] http://research.microsoft.com/en-us/projects/slayer/ (version 1.1). 
[30] https://github.com/mmjb/llvm2kittel (revision 6fc38707f7).

[31] M. Heizmann, J. Hoenicke, and A. Podelski, "Termination analysis by learning terminating programs," in Computer-Aided Verification, vol. 8559 of LNCS, pp. 797-813, Springer, 2014.

[32] http://www.netlib.org/clapack/cblas/sasum.c.

[33] S. Grebenshchikov, N. P. Lopes, C. Popeea, and A. Rybalchenko, "Synthesizing software verifiers from proof rules," in Programming Language Design and Implementation, pp. 405-416, 2012.

[34] B. Cook, A. Podelski, and A. Rybalchenko, "Termination proofs for systems code," in Programming Language Design and Implementation, pp. 415-426, ACM, 2006.

[35] W. Lee, B.-Y. Wang, and K. Yi, "Termination analysis with algorithmic learning," in Computer-Aided Verification, pp. 88-104, 2012.

[36] A. Podelski and A. Rybalchenko, "Transition invariants," in Logic in Computer Science, pp. 32-41, IEEE Computer Society, 2004.

[37] M. Heizmann, J. Hoenicke, J. Leike, and A. Podelski, "Linear ranking for linear lasso programs," in Automated Technology for Verification and Analysis, pp. 365-380, 2013.

[38] B. Cook, D. Kroening, P. Rümmer, and C. M. Wintersteiger, "Ranking function synthesis for bit-vector relations," in Tools and Algorithms for the Construction and Analysis of Systems, vol. 6015 of LNCS, pp. 236250, Springer, 2010

[39] C. David, D. Kroening, and M. Lewis, "Unrestricted termination and non-termination arguments for bit-vector programs," in European Symposium on Programming, pp. 183-204, 2015.

[40] S. Magill, M.-H. Tsai, P. Lee, and Y.-K. Tsay, "Automatic numeric abstractions for heap-manipulating programs," in Principles of Programming Languages, pp. 211-222, 2010.

[41] J. Berdine, B. Cook, D. Distefano, and P. W. O'Hearn, "Automatic termination proofs for programs with shape-shifting heaps," in $C A V$, pp. 386-400, 2006.

[42] K. L. McMillan, "Quantified invariant generation using an interpolating saturation prover," in Tools and Algorithms for the Construction and Analysis of Systems, vol. 4963 of LNCS, pp. 413-427, Springer, 2008.

[43] A. Podelski, I. Schaefer, and S. Wagner, "Summaries for while programs with recursion," in European Symposium on Programming, vol. 3444 of LNCS, pp. 94-107, Springer, 2005.

[44] E. M. Clarke, O. Grumberg, S. Jha, Y. Lu, and H. Veith, "Counterexample-guided abstraction refinement," in Computer-Aided Verification, vol. 1855 of LNCS, pp. 154-169, Springer, 2000

[45] F. Ranzato, O. Rossi-Doria, and F. Tapparo, "A forward-backward abstraction refinement algorithm," in Verification, Model Checking, and Abstract Interpretation, vol. 4905 of LNCS, pp. 248-262, Springer, 2008.

[46] M. Bozga, R. Iosif, and F. Konecný, "Deciding conditional termination," in Tools and Algorithms for the Construction and Analysis of Systems, vol. 7214 of LNCS, pp. 252-266, Springer, 2012.

[47] P. Ganty and S. Genaim, "Proving termination starting from the end," in Computer-Aided Verification, vol. 8044 of LNCS, Springer, 2013.

[48] D. Massé, "Policy iteration-based conditional termination and ranking functions," in Verification, Model Checking, and Abstract Interpretation, vol. 8318 of $L N C S$, Springer, 2014.

[49] C. Urban and A. Miné, "A decision tree abstract domain for proving conditional termination," in Static Analysis Symposium, vol. 8723 of LNCS, Springer, 2014.

[50] P. Ganty, R. Iosif, and F. Konecný, "Underapproximation of procedure summaries for integer programs," in Tools and Algorithms for the Construction and Analysis of Systems, vol. 7795 of LNCS, pp. 245-259, Springer, 2013.

[51] A. Gupta, T. A. Henzinger, R. Majumdar, A. Rybalchenko, and R.G. Xu, "Proving non-termination," in Principles of Programming Languages, pp. 147-158, ACM, 2008.

[52] H. Y. Chen, B. Cook, C. Fuhs, K. Nimkar, and P. W. O'Hearn, "Proving nontermination via safety," in Tools and Algorithms for the Construction and Analysis of Systems, pp. 156-171, 2014.

[53] T. C. Le, S. Qin, and W. Chin, "Termination and non-termination specification inference," in Conference on Programming Language Design and Implementation, pp. 489-498, 2015.

[54] W. R. Harris, A. Lal, A. V. Nori, and S. K. Rajamani, "Alternation for termination," in Static Analysis Symposium, pp. 304-319, 2010.

[55] R. Wilhelm, J. Engblom, A. Ermedahl, N. Holsti, S. Thesing, D. Whalley, G. Bernat, C. Ferdinand, R. Heckmann, T. Mitra, F. Mueller, I. Puaut, P. Puschner, J. Staschulat, and P. Stenström, "The Worst-case Execution Time Problem-Overview of Methods and Survey of Tools," Transactions on Embedded Computing Systems, vol. 7, no. 3, 2008.

[56] C. Alias, A. Darte, P. Feautrier, and L. Gonnord, "Multi-dimensional rankings, program termination, and complexity bounds of flowchart programs," in Static Analysis Symposium, vol. 6337 of LNCS, pp. 117133, Springer, 2010.

[57] M. Brockschmidt, F. Emmes, S. Falke, C. Fuhs, and J. Giesl, "Alternating runtime and size complexity analysis of integer programs," in Tools and Algorithms for the Construction and Analysis of Systems, vol. 8413 of LNCS, pp. 140-155, Springer, 2014.

[58] M. Sinn, F. Zuleger, and H. Veith, "A simple and scalable static analysis for bound analysis and amortized complexity analysis," in ComputerAided Verification, vol. 8559 of LNCS, pp. 745-761, Springer, 2014.

[59] S. Gulwani, S. Jain, and E. Koskinen, "Control-flow refinement an progress invariants for bound analysis," in Programming Language Design and Implementation, pp. 375-385, 2009.

[60] H. Y. Chen, S. Mukhopadhyay, and Z. Lu, "Control flow refinement and symbolic computation of average case bound," in Automated Technology for Verification and Analysis, pp. 334-348, 2013.

[61] C. B. Jones, "Tentative steps toward a development method for interfering programs," ACM Trans. Program. Lang. Syst., vol. 5, pp. 596-619, Oct. 1983

[62] B. Cook, A. Podelski, and A. Rybalchenko, "Proving thread termination," in Programming Language Design and Implementation, pp. 320330, ACM, 2007.

[63] A. Gupta, C. Popeea, and A. Rybalchenko, "Predicate abstraction and refinement for verifying multi-threaded programs," in Principles of Programming Languages, pp. 331-344, ACM, 2011.

[64] C. Popeea and A. Rybalchenko, "Compositional termination proofs for multi-threaded programs," in Tools and Algorithms for the Construction and Analysis of Systems, vol. 7214 of LNCS, pp. 237-251, Springer, 2012. 